\title{
Evaluation of myofibroblasts in superficial and deep layers of oral squamous cell carcinoma; an immunohistochemical study
}

\author{
Seyed Hosein Tabatabaei ${ }^{\circledR}{ }^{\oplus}$, Laleh Maleki ${ }^{2 *}{ }^{*}$ Arezoo Amirkafi $^{3 \odot}$ \\ 'Department of Oral and Maxillofacial Pathology, Social Determinants of Oral Health Research Center, School of Dentistry, \\ Shahid Sadoughi University of Medical Sciences, Yazd, Iran \\ ${ }^{2}$ Dental Research Center, Department of Oral and Maxillofacial Pathology, Dental Research Institute, Isfahan University of \\ Medical Sciences, Isfahan, Iran \\ ${ }^{3}$ Shahid Sadoughi University of Medical Sciences, Yazd, Iran
}

*Correspondence to

Laleh Maleki,

Email: I.maleki@dnt.mui.ac.ir, malekilaleh@yahoo.com

Received 5 November 2018 Accepted 15 March 2019 Published online 6 April 2019

Keywords: Oral squamous cell carcinoma, Myofibroblast, Immunohistochemistry
Citation: Tabatabaei SH Maleki L, Amirkafi A. Evaluation of myofibroblasts in superficial and deep layers of oral squamous cell carcinoma; an immunohistochemical study. Immunopathol Persa. 2019;5(1):e12. DOI:10.15171 ipp. 2019.12

\section{Abstract}

Introduction: Oral squamous cell carcinoma (OSCC) is the most common malignancy of oral cavity with a high mortality rate. Myofibroblast in the stroma of malignant tumor is one of the main factors that accelerates and modulates tumor progression and invasiveness.

Objectives: The current study aimed to investigate the presence of myofibroblasts in reactive oral lesion and OSCC and to compare its staining in superficial and deep layers in different histological grades.

Patients and Methods: The study included the archival tissues of 30 OSCCs and 30 oral reactive lesions. The myofibroblast was assessed in superficial and deep layers by immunohistochemical study of alpha smooth muscle actin ( $\alpha-S M A)$. Data were analyzed by SPSS software using chi-square, Kruskal-Wallis, and MannWhitney $U$ tests. $P<0.017$ was considered to be statistically significant.

Results: The results revealed that presence of myofibroblasts was significantly higher in OSCCs compared to oral reactive lesions $(P=0.0001)$. The results also showed that myofibroblasts presented more in the deep layers than in the superficial layers of $\operatorname{OSCC}(P=0.0001)$. A statistically significant difference was observed in myofibroblasts among different histological grades of oral squamous cell carcinoma $(P=0.0001)$.

Conclusion: The findings highlight that the presence of myofibroblast in the stroma, assessed by $a-S M A$, indicates tumor progression and invasiveness in the patients with OSCC.

\section{Introduction}

Oral squamous cell carcinoma (OSCC) is the most frequent type of oral cancers that involves more than $90 \%$ of the head and neck malignancies (1). Squamous cell carcinoma (SCC) may be developed in areas of leukoplakia and malignant cells spread into deeper tissues as the cancer develops. Men are affected more than women. Additionally, various carcinogens such as tobacco, alcohol, and human papillomavirus (HPV) increase the risk of OSCC (2).

The prediction of OSCC is dependent mainly upon the clinical stage (TNM classification), localization in the oral cavity, mode of invasion, and invasive tumor front grade (3). OSCC includes originates in the tongue, floor of the mouth, alveolar ridges, lips, buccal mucosa, retromolar trigone and hard plate (4).

Stroma of malignant tumor is one of the main factors that accelerates and modulates

\begin{abstract}
Key point
In a study on the archival tissues of 30 oral squamous cell carcinoma (OSCC) and 30 oral reactive lesions, we found the presence of myofibroblast in stroma, as assessed by alpha smooth muscle actin ( $\alpha$-SMA), indicating tumor progression and invasiveness in patients with OSCC.
\end{abstract}

tumor progression and invasiveness (5). Stroma of cancer has a specific and activated type of fibroblast named cancerassociated fibroblast or myofibroblast (6). Myofibroblasts have the characteristics of fibroblasts and smooth muscle cells. Myofibroblasts play a pivotal role in tumor progression via secretion of various cytokines (7).

Numerous molecular markers have been documented to be involved in the tumor microenvironment (8). Different markers are used for detection of myofibroblasts in

\footnotetext{
Copyright (C) 2019 The Author(s); Published by Nickan Research Institute. This is an open-access article distributed under the terms of the Creative Commons Attribution License (http://creativecommons.org/licenses/by/4.0), which permits unrestricted use, distribution, and reproduction in any medium, provided the original work is properly cited.
} 
tumor microenvironment, but alpha smooth muscle actin ( $\alpha$-SMA) is the most reliable one (9).

Few studies have evaluated the existence of myofibroblasts in OSCC and the possible role of myofibroblasts in tumor invasion(10).

\section{Objectives}

The purpose of the current study was to investigate the presence of myofibroblast in oral reactive lesions and OSCC. Accordingly, this study set out to compare the presence of myofibroblast in the superficial and deep layers of OSCC as a marker of tumor invasiveness in different microscopic grades.

\section{Patients and Methods}

\section{Sample selection}

Based on the prevalence of OSCC, the number of samples was calculated using statistical formula. A total of 60 formalin-fixed paraffin-embedded tissue blocks consisting of histopathologically diagnosed cases of inflammatory fibrous hyperplasia $(\mathrm{n}=16)$, peripheral giant cell granuloma $(n=6)$, irritation fibroma $(n=8)$, and OSCC $(n=30)$ were retrieved from the archives of the department of oral and maxillofacial pathology, Shahid Sadoughi University of Medical Sciences (from 2016 to 2017). Hematoxylin and eosin (H\&E) slides of all samples were confirmed by two expert pathologists simultaneously. Then, tissues in paraffin blocks were provided from the pathology department and sections in $4 \mu \mathrm{m}$ thickness were obtained for immunohistochemical procedures.

\section{Staining procedure}

The sections were processed for subsequent immunohistochemical study using mouse monoclonal alpha smooth muscle actin antibody (clone 1A4; DAKO, Denmark) according to the manufacturer's instructions [as described by Etemad-Moghadam et al (11)]. The sections were deparaffinized at $60^{\circ} \mathrm{C}$ for 1 hour. The sections were dewaxed in xylene and rehydrated in descending grades of alcohol. Endogenous peroxidase was reduced by incubating with $6 \%$ hydrogen peroxide for 10 minutes. After that, the specimens were washed with phosphate-buffered saline (PBS). Next, protein blocking agents were used, and all sections were incubated with primary antibody a-SMA (1:100) for 60 minutes. After washing in PBS, the slides were stained with streptavidin-biotin peroxidase kit (DAKO- Denmark), followed by rinsing with PBS and diaminobenzidine chromogen. The specimens were finally counterstained with Mayer's hematoxylin for 30 seconds, dehydrated, and finally mounted. The negative and positive tissue controls were included into each immunohistochemical process. Positive controls for SMA were obtained from the normal colon tissue.

\section{Immunohistochemical analysis}

The percentage of positive non-inflammatory and non- endothelial cells nearby the carcinomatous islands in stroma was recorded as labeling index (LI). LI was subjectively scored according to the extent of stromal positivity using the method presented by EtemadMoghadam et al (11) as follows:

Score 0: Negative or non-reactive Score 1: 1-33\% positive tumor cell Score 2: $34-66 \%$ positive tumor cell Score $3: 67-100 \%$ positive tumor cell

Staining intensity was not considered because all the stained samples had the same intensity. The percentage of immune-positive cells was also analyzed in the superficial and deep layers of the specimens.

\section{Ethical issues}

The study was conducted in accordance with the Tenets of the Declaration of Helsinki. Written informed consent was obtained from the patients to use their sample for study purposes. This research was conducted as a thesis in general dentistry by Arezoo Amirkafi and (Thesis \# 834), approved by the Committee of Ethics in Human Research at Shahid Sadoughi University of Medical Sciences, Yazd, Iran (IR.SSU.REC.1396.27).

\section{Statistical analysis}

All the parameters were assessed for statistical significance using SPSS software (version 23.0). The percentage differences of immune-positive cells between groups and superficial and deep layers were statistically analyzed. Kruskal-Wallis and Mann-Whitney $U$ tests were used for analysis of data. Additionally, a $P$ value $<0.017$ was considered to be statistically significant.

\section{Results}

The samples of this study were selected from the patients with definitive diagnosis of OSCC (30 samples) and reactive oral lesions without any dysplastic or neoplastic changes (30 samples) and compared by immunohistochemistry study of -aSMA. The specimens were obtained from the department of oral and maxillofacial pathology, Shahid Sadoughi University of Medical Sciences, Yazd, Iran. According to Table 1, the patients' age ranged from 22 to 86 years with an average of 54.23 years. Table 1 shows that OSCC was more common in males than females. Furthermore, tongue was the most affected part with mostly grade II (Table 1).

The results showed a significant difference between OSCC and control groups in the percentage of myofibroblast stained cells $(P=0.0001)$. Myofibroblasts in the control group were found to be negligible (Table 2). The results also showed a statistically significant difference between the superficial and deep layers of OSCC in terms of the presence of myofibroblasts $(P=0.0001$; Table 2$)$.

The results also showed that most of the specimens had scores 1 and 2 in the superficial layers of OSCC. In contrast, most of the specimens had score 3 in the deep 
Table 1. Distributive frequency based on age, sex, location and histological grading

\begin{tabular}{llcc}
\hline \multirow{2}{*}{ Parameters } & & \multicolumn{2}{c}{ Distributive frequency } \\
\cline { 3 - 4 } & & No. & $\%$ \\
\hline \multirow{3}{*}{ Age (years) } & $1-20$ & 0 & 0 \\
& $21-40$ & 6 & 20 \\
& $41-65$ & 18 & 60 \\
Gender & $>65$ & 6 & 20 \\
\hline \multirow{3}{*}{ Location } & Female & 13 & 43 \\
& Male & 17 & 57 \\
\hline \multirow{3}{*}{ Histological grading } & Tongue & 20 & 66.6 \\
& Lip & 2 & 6.6 \\
& Others & 8 & 26.8 \\
& I & 10 & 33.3 \\
& III & 14 & 46.7 \\
\hline
\end{tabular}

Table 2. Comparison of mean $\pm \mathrm{SD}$, minimum and maximum of myofibroblasts based on labeling index (LI) in superficial and depth of OSCC and control lesions

\begin{tabular}{llcccc}
\hline \multirow{2}{*}{ Group } & & \multicolumn{4}{c}{ Labeling index } \\
\cline { 3 - 6 } & & Mean (\%) & SD & Min. (\%) & Max. (\%) \\
\hline \multirow{2}{*}{ OSCC } & Surface & 36.96 & 15.65 & 15 & 70 \\
& Depth & 61.15 & 23.37 & 25 & 92 \\
\multirow{2}{*}{ Control } & Surface & 0.2 & 0.76 & 0 & 3 \\
& Depth & 0.06 & 0.25 & 0 & 1 \\
\hline
\end{tabular}

OSCC, Oral squamous cell carcinoma

Kruskal-Wallis test $(P=0.0001)$.

layers of OSCC $(P=0.0001$; Table 3$)$. Hence, the highest percentage of myofibroblasts (score 3 ) was seen at the deep end of OSCC (Table 3).

To compare myofibroblasts in the superficial layers of OSCC based on histological grading, most of OSCCs with grade I had scorel and those with grade II and III had score 2 (Table 4 ).

To compare myofibroblasts in the deep layers of OSCC based on histological grading, we found most of OSCCs with grade I had score 1, while grade II and III OSCCs had score 3 (Table 5). Thus, it can be said that the higher grade of OSCC had more myofibroblasts, especially in the deeper layers (Figure 1).

\section{Discussion}

OSCC is an important health problem worldwide, particularly in the developing countries. Despite the prior concept about tumor stromas that they have just a supportive role in the spread of malignancy, recent studies have shown that they are one of the predominant indicators of tumor progression and metastasis (2). Myofibroblasts are one of the most important stromal cells that affect tumor metastasis and invasion through secretion of numerous growth factors and inflammatory mediators. a-SMA-positive myofibroblasts are the hallmark of tumor-
Table 3. Comparison of frequency of myofibroblasts based on scores in superficial and depth of OSCC and control lesions

\begin{tabular}{llcccc}
\hline \multirow{2}{*}{ Group } & & \multicolumn{4}{c}{ Score } \\
\cline { 3 - 6 } & & \multirow{2}{*}{ No Stain } & $\begin{array}{c}\mathbf{1} \\
\text { No. (\%) }\end{array}$ & $\begin{array}{c}\mathbf{2} \\
\text { No. (\%) }\end{array}$ & $\begin{array}{c}\mathbf{3} \\
\text { No. (\%) }\end{array}$ \\
\hline \multirow{2}{*}{ OSCC } & Surface & $0(0)$ & $14(46.6)$ & $15(50)$ & $1(3.4)$ \\
& Depth & $0(0)$ & $8(26.6)$ & $6(20)$ & $16(53.4)$ \\
\multirow{2}{*}{ Control } & Surface & $28(93.3)$ & $2(6.7)$ & $0(0)$ & $0(0)$ \\
& Depth & $28(93.3)$ & $2(6.7)$ & $0(0)$ & $0(0)$ \\
\hline
\end{tabular}

OSCC, Oral squamous cell carcinoma

Kruskal-Wallis test $(P=0.0001)$.

Table 4. Comparison of frequency of positive $\alpha$-SMA (score) in superficial layers of OSCC based on histological grading

\begin{tabular}{lccccc}
\hline \multirow{2}{*}{ Surface } & \multicolumn{3}{c}{ OSCC Grade } & Total \\
\cline { 2 - 5 } & & $\begin{array}{c}\text { I } \\
\text { No. }(\%)\end{array}$ & $\begin{array}{c}\text { II } \\
\text { No. }(\%)\end{array}$ & $\begin{array}{c}\text { III } \\
\text { No. }(\%)\end{array}$ & No. $(\%)$ \\
\hline OSCC & 1 & $10(71.4)$ & $3(21.4)$ & $1(7.2)$ & $14(100)$ \\
score & 2 & $0(0)$ & $10(66.7)$ & $5(33.3)$ & $15(100)$ \\
& 3 & $0(0)$ & $1(100)$ & $0(0)$ & $1(100)$ \\
\hline Total & & $10(33.3)$ & $14(46.7)$ & $6(20)$ & $30(100)$ \\
\hline
\end{tabular}

OSCC, Oral squamous cell carcinoma

Kruskal-Wallis test $(P=0.0001)$.

Table 5. Comparison of frequency of positive $\alpha$-SMA (score) in deep layers of OSCC based on histological grading

\begin{tabular}{lccccc}
\hline \multirow{2}{*}{ Depth } & \multicolumn{3}{c}{ OSCC Grade } & Total \\
\cline { 2 - 5 } & & $\begin{array}{c}\text { I } \\
\text { No. }(\%)\end{array}$ & $\begin{array}{c}\text { II } \\
\text { No. }(\%)\end{array}$ & $\begin{array}{c}\text { III } \\
\text { No. }(\%)\end{array}$ & \\
\hline OSCC & 1 & $7(87.5)$ & $1(12.5)$ & $0(0)$ & $8(100)$ \\
score & 2 & $3(50)$ & $3(50)$ & $0(0)$ & $6(100)$ \\
& 3 & $0(0)$ & $10(62.5)$ & $6(37.5)$ & $16(100)$ \\
\hline Total & & $10(33.3)$ & $14(46.7)$ & $6(20)$ & $30(100)$ \\
\hline
\end{tabular}

OSCC, Oral squamous cell carcinoma.

Kruskal-Wallis test $(P=0.0001)$.

promoting cancer-associated fibroblasts (12).

The findings of the present study suggested a significant difference between OSCC and reactive oral lesions in myofibroblast staining. This finding highlights the importance of myofibroblasts in tumor development. The present finding also supports the study of Prasad et al which showed that the mean staining scores in welldifferentiated OSCC and poorly differentiated OSCC were 2.88 and 2.55 , respectively, indicating a significant difference compared to the normal control group. They concluded that malignant epithelium induced the nearby stroma to produce myofibroblast, while their proliferation could be used as a marker of malignancy (13).

Their study showed that the number of myofibroblasts in oral reactive lesions was negligible. They also found lack of myofibroblasts in oral reactive lesions and their appearance in OSCC indicated that the malignant epithelium might have a positive inductive influence on the nearby stroma for myofibroblastic differentiation. Similarly, Gupta et al used a-SMA to detect myofibroblast 

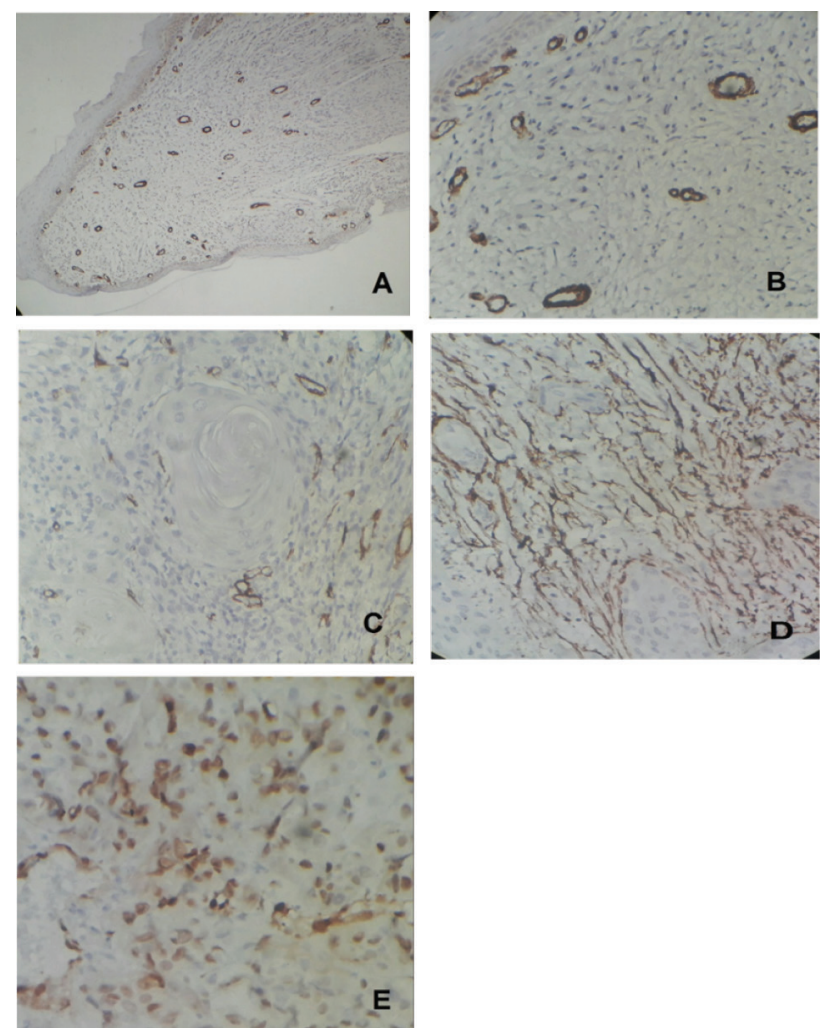

Figure 1. A; $\alpha-S M A$ staining of control group, $\times 100$. B; $\alpha-S M A$ staining of control group, $\times 400$. (C); $\alpha$-SMA staining of OSCC, $\times 400$ (well differentiated: grade I). D; $\alpha$-SMA staining of OSCC $\times 400$ (moderately differentiated: grade II). E; $\alpha$-SMA staining of OSCC $\times 400$ (poorly differentiated: grade III).

in different oral lesions. They found that normal oral epithelium was devoid of myofibroblasts. In contrast, the frequencies oral leukoplakia, oral sub-mucous fibrosis, and OSCC were $0.6 \pm 0.2(0-2), 1.2 \pm 0.68$ (1-2), and 2.6 \pm 1.34 (0-4), respectively, which showed the results were statistically significant. They concluded that myofibroblast induced tumor invasion in OSCC. Thus, the presence of myofibroblast is a prognostic marker and a therapeutic target (14).

The results of our study also suggested a significant difference in stained myofibroblasts in superficial and deep layers of OSCC. This finding indicates that the myofibroblasts in the stroma of OSCCs are crucial for the invasion of epithelial cells into the depth of oral mucosa. The study of Almangush et al also demonstrated that depth of invasion and tumor budding were concomitant with poor prognosis in individuals with early oral tongue SCC. However, cancer-associated fibroblast density and histologic risk-assessment score did not affect the survival rate (15). Interestingly, their recent finding is contrary to their previous study, where they showed that cancerassociated fibroblasts were a poor prognostic factor in tongue SCC and were strongly associated with elevated mortality (16). They argued that this difference might be due to the inclusion of individuals with stages III and IV of oral SCC of tongue in their earlier study.

Furthermore, Sindhu et al showed that depth of invasion at tumor invasive front had a statistically significant correlation with progressive histological grades of OSCC, thus showing their potential role in predicting both tumor aggressiveness and tumor prognosis. They concluded that depth of invasion could be used in the selection of patients needing more extended treatment and further lymph node assessment (17).

Consistent with the results of our investigation, the presence of stromal myofibroblasts was statistically different in grades I, II, and III of OSCC. Contrary to the present study, Parasad et al, Etemad Moghadam et al, and Ganesan $\mathrm{K}$ et al did not observed any significant difference in the distribution of myofibroblasts among different grades of OSCC (11-13).

Myofibroblasts have been recognized as the main component in the stroma of many solid malignant tumors such as breast cancer (18), prostate cancer (19), and hepatocellular carcinomas (20). However, few studies published in recent years have evaluated the presence of myofibroblasts in OSCC and the possible role of myofibroblasts in tumor invasion (10).

\section{Conclusion}

The present study was designed to determine the effect of myofibroblasts on tumor development and invasion and to evaluate the presence of myofibroblasts in the superficial and deep layers of OSCC. The findings highlight that the presence of myofibroblasts in the stroma, assessed by a-SMA, indicates tumor progression and invasiveness in patients with OSCC.

\section{Limitations of the study}

The major limitation of our research is a small sample size in each microscopic grade due to problems in providing blocks with suitable and sufficient tissues.

Conflicts of interest

No conflict of interests declared.

\section{Ethical considerations}

Ethical issues (including plagiarism, data fabrication, and double publication) have been completely observed by the authors.

\section{Authors' contribution}

All authors were involved in the study design, laboratory work, data analysis, preparation, and editing the final manuscript.

\section{Funding/Supported}

Shahid Sadoughi University of Medical Sciences supported the study.

\section{References}

1. Ferlay J, Soerjomataram I I, Dikshit R, Eser S, Mathers C, Rebelo $\mathrm{M}$, et al. Cancer incidence and mortality worldwide: sources, methods and major patterns in GLOBOCAN 2012. Int J Cancer. 2015;136:E359-386. doi: 10.1002/ijc.29210.

2. Siriwardena S, Tsunematsu T, Qi G, Ishimaru N, Kudo Y. 
Invasion-related factors as potential diagnostic and therapeutic targets in oral squamous cell carcinoma-a review. Int J Mol Sci . 2018;19:1462. doi: 10.3390/ijms19051462.

3. de Morais EF, Mafra RP, Gonzaga AKG, de Souza DLB, Pinto $L P$, da Silveira ÉJD. Prognostic factors of oral squamous cell carcinoma in young patients: a systematic review. J Oral Maxillofac Surg. 2017;75:1555-1566. doi: 10.1016/j. joms.2016.12.017

4. Markopoulos AK. Current aspects on oral squamous cell carcinoma. Open Dent J. 2012;6:126-30. doi: 10.2174/1874210601206010126

5. Jayaraj G, Sherlin H, Ramani P, Premkumar P, Natesan A. Stromal myofibroblasts in oral squamous cell carcinoma and potentially malignant disorders. Indian J Cancer. 2015;52:8792. doi: 10.4103/0019-509X.175580.

6. Bagul N, Ganjre A, Goryawala SN, Kathariya R, Dusane S. Dynamic role of myofibroblasts in oral lesions. World J Clin Oncol. 2015;6:264-71. doi: 10.5306/wjco.v6.i6.264 .

7. Sekhon HK, Sircar K, Kaur G, Marwah M. Evaluation of role of myofibroblasts in oral cancer: a systematic review. Int J Clin Pediatr Dent. 2016;9:233-9. doi: 10.5005/jpjournals-10005-1370.

8. Taghavi N, Yazdi I. Prognostic factors of survival rate in oral squamous cell carcinoma: Clinical, histologic, genetic and molecular concepts. Arch Iran Med. 2015;18:314-9. doi: 0151805/AIM.0010.

9. Mezawa $Y$, Orimo A. The roles of tumor- and metastasispromoting carcinoma-associated fibroblasts in human carcinomas. Cell Tissue Res. 2016;365:675-89. doi. org/10.1007/s00441-016-2471-1.

10. Carneiro Lúcio PS, Leite Cavalcanti A, Muniz Alves P, Pina Godoy G, Weege Nonaka CF. Myofibroblasts and their relationship with oral squamous cell carcinoma. Braz J Otorhinolaryngol. 2013;79:112-8. doi: 10.5935/18088694.20130019.

11. Etemad-Moghadam S, Khalili M, Tirgary F, Alaeddini $M$. Evaluation of myofibroblasts in oral epithelial dysplasia and squamous cell carcinoma. J Oral Pathol Med. 2009;38:63943. doi.wiley.com/10.1111/j.1600-0714.2009.00768.x.
12. Ganesan K, Nirmal Rm, Nassar Mm, Veeravarmal V, Amsaveni $\mathrm{R}$, Kumar A. Evaluation of myofibroblasts in oral squamous cell carcinoma using $\mathrm{H} 1$ calponin: An immunohistochemical study. J Oral Maxillofac Pathol. 2015;19:42.

13. Prasad BV, Kakatkar GS, Jain P, Jain M, Patel M, Khan J. Expression of myofibroblasts in oral squamous cell carcinoma: an immunohistochemical study. J Contemp Dent Pract. 2016;17:857-60.

14. Gupta K, Metgud R, Gupta J. Evaluation of stromal myofibroblasts in oral leukoplakia, oral submucous fibrosis, and oral squamous cell carcinoma - an immunohistochemical study. J Cancer Res Ther. 2015;11:893-8.

15. Almangush A, Bello IO, Keski H, Antti S, Akinen LKM, Kauppila $\mathrm{JH}$, et al. Depth of invasion, tumor budding, and worst pattern of invasion: Prognostic indicators in early-stage oral tongue cancer. Head Neck. 2013;36:811-8. doi: 10.1002/hed.23380.

16. Bello IO, Vered M, Dayan D, Dobriyan A, Yahalom R, Alanen $\mathrm{K}$, et al. Cancer-associated fibroblasts, a parameter of the tumor microenvironment, overcomes carcinoma-associated parameters in the prognosis of patients with mobile tongue cancer. Oral Oncol. 2011;47:33-8.

17. Sindhu G, Swati B, Shashikant K, Pratima K, Vinay H, Suchitra G. Depth of invasion and GLUT-1 as risk predictors in oral squamous cell carcinoma: an immunohistochemical study. Translational Research in Oral Oncology. 2017;2:1-10. doi. org/10.1177/2057178X16689690.

18. Catteau X, Simon P, Noël J-C. Myofibroblastic stromal reaction and lymph node status in invasive breast carcinoma: possible role of the TGF- $\beta 1 /$ TGF- $\beta$ R 1 pathway. BMC Cancer. 2014;14:499.

19. Palethorpe HM, Leach DA, Need EF, Drew PA, Smith E. Myofibroblast androgen receptor expression determines cell survival in co-cultures of myofibroblasts and prostate cancer cells in vitro.Oncotarget. $2018 ; 9: 19100-14$. doi: 10.18632/ oncotarget.24913.

20. Affo S, Yu L-X, Schwabe RF. The Role of Cancer-Associated Fibroblasts and Fibrosis in Liver Cancer. Annu Rev Pathol. 2017;12:153-86. 